\title{
Produção CIENTífica SOBRe PSICOTERAPIAS NA BASE de DADOS PePSIC (1998/2007)
}

\author{
Fabiana Pessini Pinto ${ }^{\star}$ \\ Tales Vilela Santeiro ${ }^{\star}$ \\ Fabiola Ribeiro de Moraes Santeiro ${ }^{\star \star \star}$
}

\begin{abstract}
Resumo
Esta pesquisa objetiva analisar a produção científica publicada no período entre 1998 e 2007 sobre psicoterapia, considerando: distribuição das publicações ano a ano; periódicos; quantidade e sexo dos autores; tipos de pesquisa; enfoque teórico das produções; afiliação institucional dos autores e estratégias de avaliação psicológica utilizadas, material constituído de artigos publicados em periódicos cientificos indexados à base de dados PePsic $(N=117)$, obtido com o verbete "psicoterapia". A análise apresentada é quantitativa. Os autores das produções são do sexofeminino em sua maioria. Dos tipos de trabalhos analisados, houve predomínio de teóricos, seguidos por empíricos. Cinco dos periódicos possuem 50,5\% da produção pesquisada. O enfoque teórico psicanalítico teve maior predominância, seguido do cognitivo e comportamental. A maior parte dos autores está afiliada a instituições brasileiras, as quais estão concentradas na região sudeste do país. Houve uma maior prevalência para o uso de entrevistas e escalas como estratégias de avaliação psicológica.
\end{abstract}

Palavras-chave: psicologia clínica; modelos teóricos; metaciência; literatura cientifica; discurso científico.

\section{SCIENTIFIC PRODUCTION CONCERNING PSYCHOTHERAPIES in PePSIC databases (1998/2007)}

\footnotetext{
Abstract

On main purpose of scientific production analysis, published in the period between 1998-2007, on the subject of psychotherapy, take into consideration: distribution of publications year by year; regular advisers; quantity and gender of authors; types of research; focus on the theory of the productions; institutional

^ Psicóloga. Graduada pela Universidade de Franca.

E-mail: fapepi@yahoo.com.br

$\star \star$ Psicólogo. Doutor em Psicologia como Profissão e Ciência pela Pontifícia Universidade Católica de Campinas. Atualmente é Professor Efetivo - Adjunto, Nível 1, da Universidade Federal de Goiás/Campus Avançado de Jataí. Endereço: Universidade Federal de Goiás. Rua Riachuelo, 1.530 - Samuel Graham. Jataí, GO - Brasil - Caixa-Postal: 03. CEP: 75804-020.

E-mail: talessanteiro@hotmail.com

$\star \star \star$ Psicóloga. Graduada pela Universidade de Franca.

E-mail: fabiolarmsanteiro@hotmail.com
} 
affiliation of the authors and strategies for psychological evaluation. The body is constituted by articles published in scientific periods, indexed to the PePsic databases $(N=117)$ was obtained by the time the word "psychotherapy" was used. The analysis presented is quantitative. Authors of the productions are, mainly, the feminine sex. There were a higher number of theoretical works. Five from the regular advisers own 50.5\% of the entirely searched production. Psychoanalytic approach had more theoretical dominance. Most authors are affiliated to Brazilian institutions, which are concentrated in the southeast region. There were a higher prevalence for the use of interviews and scales as assessments strategies.

Keywords: clinical psychology; theoretical models; metascience; scientific literature; scientific speech.

\section{INTRODUÇão}

A atuação do psicólogo tem sido conhecida pelo seu amplo campo de trabalho, dentre as diversas profissões e áreas afins. A mais clássica ou a que mais é lembrada quando se pensa em Psicologia é a psicoterapia, uma das atividades possíveis na área de atuação clínica. Alguns autores conceituam as psicoterapias como "[...] métodos de tratamento para os problemas emocionais" (SOUZA; TEIXEIRA, 2004, p. 46), através dos quais um profissional treinado auxilia aquele que pede ajuda e estabelece assim uma relação profissional. Usualmente essa relação tem com objetivos remover ou modificar comportamentos ou sintomas existentes, prevenir sua aparição e em última instância promover crescimento pessoal, bem como o desenvolvimento da personalidade.

A psicoterapia, em sua forma mais fundamental, existe muito antes da Psiquiatria ou de qualquer noção sistemática de tratamento psicológico; ela envolve um sistema de relação humana em que duas pessoas se encontram: uma delas necessitada de ajuda emocional, moral ou religiosa, e outra com características pessoais e atributos que possam solucionar o problema, geralmente representadas, respectivamente, como suplicante e curandeiro (STONE, 2005).

Desde a Antiguidade a esse curandeiro foi dada a noção de alguém que possui qualidades para "iluminar", sendo que essa noção existe em várias culturas, por exemplo, nas orientais, como japonesas e chinesas. A idéia de levar a luz para onde antes havia escuridão é naturalmente preservada nas linguagens românticas do francês e também do português. A diferença da psicoterapia da Antiguidade e ao que atribuímos com suas características 110 contemporâneas reside na falta de participação ativa entre paciente-terapeuta, práticas pelas quais não exigiam naquele período a auto-revelação do paciente, como a crença da cura pela imposição das mãos (STONE, 2005). 
Messer e Wachtel (1997) indicam que a diferença entre as diversas formas de prestar ajuda ao longo do tempo está essencialmente no fato da 'apropriação' das práticas religiosas pelas ciências. Ou seja, apesar das práticas serem semelhantes, as psicoterapias são consideradas científicas, o que as remete a outra ordem de saberes, o que gerou as diversas formas de psicoterapia na Modernidade.

Nesse sentido, a psicoterapia tem suas raízes históricas na Medicina antiga, na religião, na cura pela fé e no hipnotismo, mas começou a ser utilizada para tratamento de doenças nervosas e mentais no final do século XIX, quando se tornou uma arte médica restrita aos psiquiatras. Foi no decorrer do século XX que outros profissionais passaram a exercê-la e hoje a psicoterapia é utilizada por psicólogos, psiquiatras, médicos clínicos, enfermeiros e assistentes sociais, sendo reconhecido o fato de ser um campo interdisciplinar (RODRIGUES; BRITO, 2009). Porém, se conservou o termo "psicoterapia"e outras terminologias de origem médica, como diagnóstico, doença, etiologia, paciente e psicoterapeuta.

A psicoterapia, portanto, em seu sentido mais amplo, envolve um tipo especial de interação única, na qual há aquele que precisa de ajuda, pois seu sofrimento é, de alguma forma, ocasionado mais pela mente do que pelo corpo, e há aquele que prestará o serviço, o qual é escolhido para auxiliar, pois se espera que, por meio de sua empatia, sabedoria e conhecimento, possa oferecer condições de ajuda para aquele que o procura, por meio do instrumento da palavra falada (STONE, 2005), embora seja sabido que alguns enfoques teóricos não se atenham a esse instrumento, apenas.

No transcurso histórico da evolução das formas de cuidado do sofrimento humano, principalmente após o advento da psicanálise, como modalidade de escuta e compreensão das manifestações humanas, até os sistemas que foram desenvolvidos abertamente por serem contrários a ela, como é o caso da psicoterapia fenomenológico-existencial, as psicoterapias têm sofrido transformações incessantes e tantos desdobramentos que são, hoje, difíceis de serem catalogados. Nesse sentido, as pesquisas científicas no campo das psicoterapias passaram a ser necessárias e desenvolvidas progressivamente para dar conta dessas evoluções e também para explicar seus próprios modos de ação, a ponto de atualmente haver centros de pesquisa em muitos países. As psicoterapias, portanto, variam segundo as técnicas, as teorias nas quais se baseiam e os objetivos que as norteiam, bem como a freqüência e o tempo de duração das sessões.

As teorias e técnicas psicoterápicas abrangem as psicoterapias breves de apoio, destinadas a auxiliar o paciente a superar dificuldades momentâneas (GRETHER; HASSENE, 2007; WEISSMAN; MARKOWITZ; KLERMAN, 2009); a terapia comportamental que utiliza exposição in vivo, a prevenção de resposta, o condicionamento clássico (COELHO, 2008; D'EL REY; PACINI, 2006); a ludoterapia infantil (CAMPOS; CURY, 2009; SEI, 2008); as terapias de grupo, de família e de casais (ALMEIDA; COSTA; GOMES, 2009; CANETE; VITALLE; SILVA, 2008; MOTTA; SANTOS, 2002); a hipnose (NEUBERN, 2004, 2006); o 
psicodrama (COSTA; DIAS, 2005; GULASSA, 2007); a psicanálise e a psicoterapia de orientação analítica que se propõem, dentre outras, a modificar aspectos da personalidade pela obtenção de insight (FINKEL, 2009; SANTEIRO, 2008).

Pelas razões apontadas, muito se discute sobre as dificuldades e desafios de se fazer pesquisa em psicoterapia (FIORINI, 1991; SANTOS; ZASLAVSKY, 2007). Este trabalho e os questionamentos inerentes a ele nascem nesse contexto e ele tem o interesse de analisar o que tem sido produzido no âmbito das psicoterapias, traçar o perfil destas e de seus pesquisadores, focalizando, de modo especial, as tendências divulgadas no país e em realidades latino-americanas circunvizinhas.

Em síntese, o objeto de pesquisa proposto diz respeito à análise de produção científica sobre psicoterapias na base de dados eletrônica PePsic, a qual será descrita mais adiante no item "Método". Enfocar o estado da pesquisa sobre um determinado tema permite,

[...] não apenas dimensionar esta área, mas também redefinir as estratégias mais pertinentes ao saber-fazer-poder, analisar o estado da arte, apontar para algumas tendências e propor parâmetros de comparação com outros ramos do saber científico (OLIVEIRA apud PACHECO, 2005, p. 9).

Para Robins, Gosling e Craik (1999), este tipo de pesquisa permite ao investigador situar-se diante do crescimento científico que tem ao seu redor (âmbitos regional, nacional ou internacional), avaliar tendências com base empírica, não apenas fundamentadas na intuição ou especulação.

Por produção científica deve-se levar em consideração toda e qualquer forma de pesquisa e produção textual desenvolvida para ganhos de progresso tecnológico, social e humano. De acordo com Oliveira Filho, Hochman e Nahas (2005), é por meio da publicação que o trabalho científico atinge seu objetivo, é ela que dá a existência ao que foi desenvolvido pelo pesquisador, na medida em que traz ao público o seu processo de produção do conhecimento, seja ele entendido a partir de quaisquer paradigmas em consideração. Ainda sobre este tema, Pacheco (2005) diz que produzir e comunicar o conhecimento pode garantir o exercício da investigação, o intercâmbio de idéias e possíveis soluções para problemas humanos. Além de "[...] contribuir para avançar o conhecimento na área e tornar acessíveis as informações produzidas, [o pesquisador] submete à crítica suas idéias, métodos e descobertas" (PACHECO, 2005, p. 9).

Por toda essa relevância vinculada à socialização do saber, logo que se dá início ao mundo acadêmico, os alunos de graduação devem estar preparados para a tarefa de produzir ciência (WITTER, C.; BURITI; WITTER, G., 2007), comunicar dados de pesquisas, tratar dados quantitativamente e/ou qualitativamente. Isso se dá em diversas áreas do conhecimento humano e não é diferente na Psicologia, na qual observar, analisar e pesquisar se faz uma constante na vida profissional e deve estar presente na formação, que se deseja imperativamente continuada. 
O trabalho intelectual do pesquisador depende da forma como é feita a comunicação com seus leitores, meios estes pelos quais os pesquisadores utilizam para informar e comunicar os resultados do que foi elaborado e pesquisado em seus estudos e também para analisar e discutir resultados alcançados por outros cientistas. Assim, toda pesquisa envolve atividades diversas de publicações que influenciam diretamente as relações entre pesquisador - resultados - leitores (WITTER, C.; BURITI; WITTER, G., 2007).

Consideradas essas breves observações sobre psicoterapias e produção científica, segue a especificação dos norteadores desse trabalho. Em termos gerais, objetivou analisar a produção científica em psicoterapia utilizando o verbete destinado à pesquisa: "psicoterapia", para a busca de artigos na base de dados eletrônica PePsic. A escolha desse verbete considerou que era suficientemente abrangente, por exemplo, incluir os comportamentalistas, uma vez que nem sempre adotam o prefixo "psico" nas suas produções, e sim "terapia" comportamental. Com isto o risco de excluir da amostra parte da produção dessa frente teórica ficou descartado.

De modo mais específico, objetivou a análise dos artigos quanto: a) distribuição de publicações ano a ano; b) tipos de trabalhos (empíricos ou outros tipos); c) periódicos; d) enfoque teórico das psicoterapias; e) etapa do desenvolvimento pesquisada (infância, adolescência, vida adulta, velhice); f) psicopatologias pesquisadas; g) afiliação institucional dos autores; h) sexo dos autores; e i) estratégias de avaliação psicológica utilizadas (entrevista, questionário, observação, escala, teste psicológico etc).

\section{MÉTodo}

\section{Material}

Foram utilizados artigos de periódicos científicos divulgados na base eletrônica de dados PePsic. Este é o meio eletrônico que reúne uma coleção de revistas cientificas em Psicologia e áreas afins. Segundo o próprio site, esta base de dados é fruto da parceria entre a Biblioteca Virtual em Saúde - Psicologia (BVS-Psi) e a Associação Brasileira de Editores Científicos de Psicologia - ABECiP. Tem como meta ampliar o acesso à produção científica em Psicologia e áreas afins, através da publicação de periódicos em formato eletrônico e sua disponibilização gratuita na Internet.

\section{Procedimento}

O material foi obtido utilizando o verbete "psicoterapia" para a pesquisa e busca de artigos na base de dados eletrônica PePsic. Inicialmente sem limitações no idioma de interface e no ano da publicação. Em seguida, após uma visão geral do total de produções, e considerar a publicação dos últimos dez anos, foi feita a opção basicamente por: 1) tratar-se de um período de tempo bastante abrangente, para analisar a produção científica em psicoterapia naquela base de dados; 2) considerar as bases de dados que disponibilizam artigos on-line como o fenômeno mais difundido nos anos 2000, embora seja sabido que em datas anteriores havia outras que disponibilizavam resumos. 
No que diz respeito à análise dos tipos dos artigos (item "b" dos objetivos específicos), foram consideradas cinco categorias distintas:

1) EE - Estudo Empírico: pesquisa envolvendo levantamento e análise de dados com a finalidade de conhecer um fenômeno ou de testar uma hipótese. Usualmente é estruturado com base nos itens: introdução, objetivos, método, resultados, discussões e conclusões e envolve a participação de seres humanos.

2) RE - Relato de Experiência: aplicação de novos programas ou formas de intervenção desenvolvidas empiricamente e que são descritas por seus autores e/ou praticantes.

3) RL - Revisão de Literatura: revisão sistemática da literatura concernente a um tema ou tópico específico com o objetivo de delinear o estado da arte, identificar principais autores e/ou pesquisas, mostrar a evolução de conhecimentos sobre um tema específico, apontar falhas e acertos dos diversos trabalhos na área, constituindo o resumo do que é realmente importante sobre o tema focalizado.

4) TT - Trabalho Teórico: voltado para a reflexão de um tema, tópico ou conceito teórico ou para a descrição de uma técnica psicoterapêutica. Exclui trabalhos teóricos com ilustrações clínicas, não se tratando, pois, de pesquisa sistemática como verificado nos RL acima.

5) TTI - Trabalho Teórico Ilustrado: voltado para a reflexão de um tema, tópico ou conceito teórico, acompanhado, necessariamente, de um ou mais casos clínicos práticos com a finalidade de ilustração.

\section{Resultados E DisCuSSÃo}

Foram encontrados 121 artigos no período considerado, sendo que cinco deles não se encaixaram no perfil proposto pelo presente trabalho. Fizeram parte desta exclusão trabalhos que não são considerados artigos, como a resenha de Oddone (2005) Suicídio e psicoterapia - uma visão gestáltica, da autora Karina Fukumitsu, divulgado no periódico Estudos e Pesquisas em Psicologia. Outro exemplo é o relato de Teixeira e Gonçalves (2005) sobre o XIV Encontro Brasileiro de Psicoterapia e Medicina Comportamental, divulgado na revista Psicologia: Teoria e Prática. Foram considerados, portanto, 117 artigos da base de dados Pepsic, os quais foram o objeto desta pesquisa.

\section{Distribuição das PublicaÇões ano a ANo}

Para compreensão contextualizada do tamanho da amostra estudada, é necessário considerar que a última busca efetuada para este trabalho na Base PePsic datou de meados 2008. As publicações analisadas estão, assim, situadas historicamente entre o ano de 1998 e 2007, sendo que a maior concentração está no ano de 2005, com 37 artigos (31,62\%), e, em segundo lugar, o ano de 2006, com 24 artigos (20,51\%) (Figura 1). Dessa forma foi percebido um aumento significante na quantidade de produções desde 1998, atingindo em 2005 um grande número de trabalhos, marcando um aumento no interesse de se pesquisar a psicoterapia, pelo menos no que diz respeito às produções indexadas à PePsic. 
Figura 1 - distribuição dos artigos ano a ano (1998/2007).

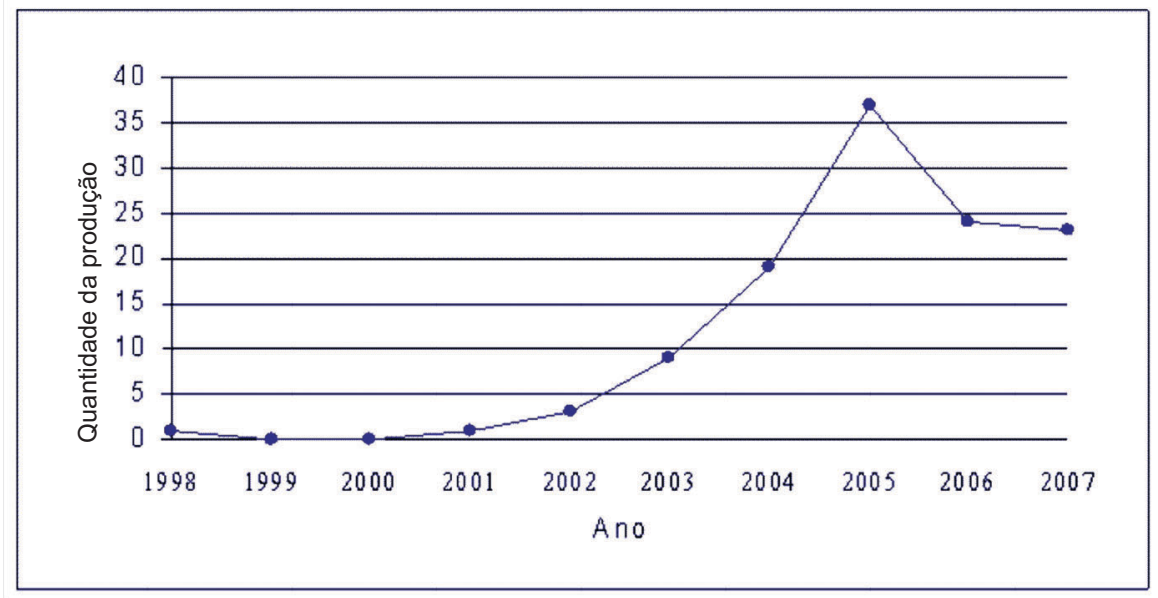

\section{TIPOS DE TRABALHO}

Os Tipos de Trabalho desenvolvidos nas produções analisadas mostra que há um maior número de Trabalhos Teóricos sendo desenvolvidos sobre psicoterapia ( $\mathrm{n}=46)$; estes estão voltados mais para a discussão de um tema, tópico ou conceito teórico, excluindo trabalhos com ilustração clínica. Trabalhos Empíricos são os que se seguiram $(n=27)$ e se tratavam de pesquisas envolvendo levantamento e análise de dados, além de envolverem participação de seres humanos.

Trabalhos Teóricos Ilustrados totalizaram 20 produções; seguidos de Relatos de Experiências $(n=19)$; Revisão de Literatura $(n=3)$ e houve aqueles trabalhos classificados como Não Especificados quanto ao Tipo, por seus textos não terem permitido a classificação dentro das categorias anteriores $(n=2)$.

\section{Periódicos}

Dos 31 periódicos encontrados na busca, a maior parte dos artigos foi divulgada na revista Psicologia: Ciência e Profissão, totalizando 17 artigos (15\%). Este órgão divulgador diz respeito a uma publicação do Conselho Federal de Psicologia (Tabela 1).

O segundo maior divulgador sobre psicoterapia na base de dados PePsic foi o periódico Estudos em Psicologia (Campinas), com um total de 13 artigos (11\%). Este é um veículo de publicação do Programa de Pós-Graduação da PUC Campinas. O terceiro maior divulgador de artigos encontrados nesta pesquisa foi o periódico Vínculo, com 12 publicações (10\%). Esta revista é uma publicação do Núcleo em Saúde Mental e Psicanálise das Configurações Vinculares. A Revista Brasileira de Terapias Cognitivas apareceu com 9 artigos (7\%), e em seguida a Revista da SPAGESP, Sociedade de Psicoterapias Analíticas Grupais do Estado 
de São Paulo, com 8 artigos (6\%). Estes cinco primeiros periódicos marcaram $50,5 \%$ dos artigos na frequência acumulada, o que foi considerado como resultado expressivo (Tabela 1).

Muitos periódicos tiveram a freqüência de apenas um artigo indexado à base de dados analisada; estes foram incluídos na categoria "outros", como é o caso do periódico Psychê, uma Publicação do Centro de Estudos e Pesquisa em Psicanálise da Universidade São Marcos, cujo artigo da autora Brum (2006) "A depressão materna e suas vicissitudes" foi exemplo encontrado nesta busca.

Tabela 1 - periódicos divulgadores.

\begin{tabular}{lcccc}
\hline \multicolumn{1}{c}{ Periódico } & F & \% & FA & FA \% \\
\hline Psicologia Ciência e Profissão & 17 & 15 & 17 & 15 \\
Estudos de Psicologia (Campinas) & 13 & 11 & 30 & 25,5 \\
Vínculo & 12 & 10 & 42 & 36 \\
Revista Brasileira de Terapias Cognitivas & 9 & 8 & 51 & 43.5 \\
Revista da SPAGESP & 8 & 7 & 59 & 50,5 \\
Psicologia USP & 7 & 6 & 66 & 56.5 \\
Psic: Revista da Vetor Editora & 6 & 5 & 72 & 61.5 \\
Psicologia: Teoria e Prática & 5 & 4 & 77 & 66 \\
Revista Brasileira de Terapia Comportamental e Cognitiva & 4 & 3.5 & 81 & 69 \\
Psicologia para a América Latina & 4 & 3,5 & 85 & 72,5 \\
Psicologia Escolar e Educacional & 3 & 2.5 & 88 & 75 \\
Psico USF & 3 & 2,5 & 91 & 77 \\
Perspectivas Psicológicas & 3 & 2,5 & 94 & 80 \\
Cógito & 3 & 2.5 & 97 & 83 \\
Estudos e Pesquisas em Psicologia & 2 & 1,7 & 99 & 84,5 \\
Diversitas & 2 & 1,7 & 101 & 86 \\
Aletheia & 2 & 1,7 & 103 & 88 \\
Outros & 14 & 12 & 117 & 100 \\
\hline Total & $\mathbf{1 1 7}$ & $\mathbf{1 0 0}$ & $\mathbf{1 1 7}$ & $\mathbf{1 0 0}$ \\
\hline
\end{tabular}

\section{ENFOQUE TEÓRICO DAS PSICOTERAPIAS}

Os resultados das produções analisadas quanto ao Enfoque Teórico apontaram a Psicanálise como o referencial prevalente no total de trabalhos, totalizando $32 \%$. Em seguida, o enfoque Cognitivo e Comportamental, com 20\% dos artigos analisados. O enfoque em Grupoanálise foi outra categoria que se destacou, com $7 \%$ dos trabalhos. Na categoria "Outros" foram englobados os artigos de enfoque teórico com menor frequência, em comparação aos apresentados anteriormente $(\mathrm{n}=19)$. Fizeram parte desta categoria os produtos de enfoque teórico: Fenomenológico-Existencial $(n=3)$, Psicoterapia Analítica $(n=3)$, Gestalt $(n=3)$, Terapia Sistêmica $(n=3)$, Construcionismo Social $(n=2)$, Psicodrama $(n=2)$, Sócio-Histórico $(n=2)$ e Logoterapia $(n=1)$.

Houve parcela dos artigos analisados na qual não foi possível identificar o Enfoque Teórico $(n=30)$. O principal fator que explica esse resultado é o fato de nesses trabalhos o objetivo principal girar em torno de psicoterapias e/ou formação de psicoterapeutas de forma ampla. Exemplo desta condição é o artigo de 
Sakamoto (2006), intitulado "Foco na estratégia da supervisão clínica em psicoterapia breve", no qual a autora discute sobre a experiência de estágio de alunos de determinado curso de Psicologia e o papel do supervisor em Psicoterapia Breve, sem, contudo, esclarecer o entendimento teórico norteador dessa psicoterapia. Não havia, portanto, pertinência em se classificar essa produção como pertencente a algum enfoque teórico específico.

\section{ETAPA do DESENVOLVIMENTO PESQUISADA}

Quanto à Etapa do Desenvolvimento dos participantes dos estudos investigados, foi levado em consideração o referencial de Papalia, Olds e Feldman (2006) para a determinação das idades e suas respectivas etapas do desenvolvimento, utilizando como critério apenas os trabalhos que apresentaram essa informação $(\mathrm{N}=42)$ : a) infância, como o período que engloba a primeira (nascimento aos 3 anos), a segunda (3 aos 6 anos) e a terceira infância (6 aos 11 anos); b) adolescência, que compreende as idades de 11 a aproximadamente 20 anos; c) vida adulta que diz respeito ao jovem adulto (20 aos 40 anos) e ao adulto de meia-idade (40 aos 65 anos); e d) terceira idade, que vai dos 65 anos até o fim da vida.

Considerando essas etapas, foi observado o predomínio significante do estudo da infância $(n=24 ; 57,0 \% ;[\chi 2(3, N=42)=24,45, p<0,001])$, seguido de estudos sobre a adolescência $(n=8 ; 19,0 \%)$ e a vida adulta $(n=7 ; 17,0 \%)$. Também foi constatada a existência de poucos trabalhos sobre a terceira idade $(n=3 ; 7 \%)$.

Desse modo, devido à relevância da infância, adolescência e velhice de per se no contexto sociocultural de um país, fica evidente a necessidade de uma maior atenção por parte dos pesquisadores da área de psicoterapia às últimas etapas de desenvolvimento. Naturalmente não se procura dizer que, no Brasil, não tenha havido iniciativas que contemplem essas fases da vida, posto que isso seria insustentável, mas somente que o identificado na PePsic e com o verbete psicoterapia foi tido como escasso e/ou que tem havido poucas publicações indexadas das atividades/ atendimentos e investigações realizadas. Esses resultados parecem caracterizar um possível campo em aberto para o profissional e pesquisador da psicoterapia.

\section{Psicopatologias pesquisadas}

Outro interesse do trabalho foi investigar as psicopatologias que têm merecido atenção dos autores na área das psicoterapias. Dos 117 artigos aqui considerados em análise, 22 (19\%) se preocupavam em discutir alguma patologia. O critério utilizado para a nomenclatura aqui estabelecida foi o próprio nome utilizado pelos autores das produções. Em seguida, foi utilizada a nomenclatura proposta por Sadock, B. e Sadock, V. (2007), os quais fazem o uso do manual de nosologia DSMIV (Manual Diagnóstico e Estatístico de Transtornos Mentais) para a classificação dos transtornos mentais. Nesse sentido, os transtornos especificados pelos autores eram alocados em grandes eixos. Por exemplo, se o transtorno investigado era a depressão, esse dado era alocado na categoria "Transtornos do Humor", e assim sucessivamente até ser completada a análise de todas as psicopatologias. 
Os Transtornos de Ansiedade foram os mais investigados ( $n=5)$; dentre eles estão o transtorno de pânico, o transtorno de ansiedade social e o transtorno de stress pós-traumático. Em seguida, foram observados os Transtornos da Alimentação $(n=4)$, como a anorexia nervosa e a bulimia nervosa. Os Transtornos de Humor se apresentaram como o terceiro grupo de patologias mais frequentemente estudado $(n=3)$, sendo esse mesmo resultado constatado, tanto para os Transtornos Relacionados a Substâncias, que dizem respeito à drogadição, quanto para os Transtornos de Personalidade. Em seguida, foram observados os Transtornos de Déficit de Atenção e Hiperatividade (n=2). O Retardo Mental e o Transtorno Global do desenvolvimento tiveram a menor frequência observada, sendo 1 estudo classificado em cada uma dessas categorias.

Sendo assim, em conformidade com a classificação adotada pelo DSM-IV, foi constado que a maioria dos transtornos aqui elencados se encontra no Eixo I, o Eixo dos transtornos clínicos e de outras condições que possam ser foco de atenção clínica. Apenas dois fazem parte do Eixo II, que são os transtornos de personalidade e o retardo mental (SADOCK, B.; SADOCK, V., 2007).

Sobre o resultado global acerca das psicopatologias, algumas observações são, ainda, necessárias. A análise detalhada das patologias mais pesquisadas, embora de extrema relevância para traçado qualitativo da produção sobre psicoterapias, mostrou-se incipiente porque, exceto nos trabalhos classificados em patologias únicas e anteriormente relatados, nos demais os autores forneciam idéia muito geral e inespecífica a esse respeito. Além do mais, a patologia não era especificada nos estudos porque não era este o objetivo dos autores de muitos deles. É imprescindível observar que, a depender da orientação teórica adotada, a própria nomenclatura "psicopatologia" não se aplicava.

\section{Afiliação institucional dos aUtores}

Sobre a afiliação dos autores foi levantado um total de 102 instituições. Por ser este número extenso serão especificadas as instituições que apresentaram o mínimo de 5 autores a elas vinculados. Dentre as instituições as quais os autores são afiliados a Faculdade de Psicologia da Universidade de Buenos Aires, está em primeiro lugar, com 15 autores. Trata-se de um resultado peculiar, associado ao artigo de Slapak, Passalilcqua, e Cervone (2002) e mais 12 autores em colaboração. Em seguida, a instituição vista na pesquisa é a Pontifícia Universidade Católica do Rio Grande do Sul (PUC-RS), com 8 autores, e, com o mesmo número de autores, a Universidade de São Paulo (USP).

Ainda sobre a afiliação, com 7 autores filiados a cada instituição estão a Universidade Estadual de Londrina e a Universidade Federal do Rio Grande do Sul. A Sociedade de Psicoterapias Analíticas Grupais do Estado de São Paulo aparece em seguida, com 6 autores filiados. E, com 5 autores cada, ficaram, ao todo, sete instituições: a Associação de Psicoterapia e Estudos Psicanalíticos, Divisão de Psicologia Clínica do IEHC - FMUSP, PUC-Campinas, Universidade 
Federal de Uberlândia, Unicamp, Universidade Católica de Goiás e a Universidade Presbiteriana Mackenzie. Demais detalhamentos sobre Instituições podem ser visualizados no Quadro 1.

Quadro 1 - relação de instituições dos autores dos estudos (ordem alfabética de instituição; continua).

\begin{tabular}{|c|c|}
\hline Instituições & $\begin{array}{c}\mathbf{N}^{\circ} \text { de } \\
\text { autores }\end{array}$ \\
\hline Ambulatório Regional de Especialidades - NGA Botucatu & 1 \\
\hline Asociación Argentina de Psicologia y Psicoterapia de Grupo & 3 \\
\hline Associação de Psicoterapia e Estudos Psicanalíticos & 5 \\
\hline Associação Brasileira de Psicoterapia & 1 \\
\hline Associação de Psicopterapia Psicanalítica Argentina & 1 \\
\hline Associação Mexicana de Psicoterapia de Grupo & 1 \\
\hline Associaçao Mexicana de Psicoterapia Psicanalítica & 1 \\
\hline Associação Mundial de Psicanálise - França & 1 \\
\hline Associação Psicoterapia Psicanalítica & 1 \\
\hline Causa Freudiana de Paris - França & 1 \\
\hline Centro de Estudos de Psicoterapia de Grupo de Franca e Região & 3 \\
\hline Centro de Estudos e Eventos Psicanalíticos de Uberlândia & 1 \\
\hline Centro de Investigación y Treinamento em Psicoterapia Gestalt & 1 \\
\hline Centro de Pesquisas e Tratamento de Transtorno de Ansiedade de São Paulo & 3 \\
\hline Centro de Psicoterapia Cognitivo - Comportamental & 2 \\
\hline Centro de Referência em Educação Especial da Secretaria da Educação do Estado de Sergipe & 1 \\
\hline Centro Universitário de Brasília & 1 \\
\hline Centro Universitário Nossa Senhora do Patrocínio & 1 \\
\hline Colégio Objetivo - Alto Padrão Franca & 1 \\
\hline Divisão de Psicologia Clínica do IEHC - FMUSP & 5 \\
\hline Escola Brasileira de Psicanálise - Bahia & 1 \\
\hline Escuela de Psicologia de La Universidad Iberoamericano & 1 \\
\hline Faculdade de Medicina de São José do Rio Preto & 1 \\
\hline Faculdade de Psicologia - Universidade de Buenos Aires & 15 \\
\hline Faculdade de Psicologia de Buenos Aires & 1 \\
\hline Faculdade Ruy Barbosa & 1 \\
\hline Federação Brasileira de Psicodrama & 1 \\
\hline Group Analytic Society - Londres & 2 \\
\hline Hospital Aristides Maltez & 1 \\
\hline Hospital das Clínicas de Porto Alegre & 1 \\
\hline Hospital do Pronto Socorro de Porto Alegre & 2 \\
\hline Hospital Psiquiátrico Lovencial & 1 \\
\hline Hospital Psiquiátrico São Pedro & 1 \\
\hline Hospital Psiquiátrico Tampico (México) & 2 \\
\hline Instituto Catarinense de Terapia Cognitiva & 1 \\
\hline Instituto de Ensino e Pesquisa em Psicoterapia & 1 \\
\hline
\end{tabular}


Quadro 1 - relação de instituições dos autores dos estudos (ordem alfabética de instituição; continuação).

\begin{tabular}{|c|c|}
\hline Instituições & $\begin{array}{c}\mathbf{N}^{0} \text { de } \\
\text { autores }\end{array}$ \\
\hline Instituto de Gestalt Terapia de Brasília & 2 \\
\hline Instituto de Psicologia Aplicada e Formação & 2 \\
\hline Instituto de Psiquiatria HC - FMUSP & 2 \\
\hline Núcleo de Estudos em Saúde Mental e Psicanálise das Configurações vinculares & 3 \\
\hline Núcleo de Oncologia da Bahia & 1 \\
\hline Pontifícia Universidade Católica de Campinas & 5 \\
\hline Pontifícia Universidade Católica de São Paulo & 1 \\
\hline Pontifícia Universidade Católica do Rio de Janeiro & 3 \\
\hline Pontifícia Universidade Católica do Rio Grande do Sul & 8 \\
\hline Prefeitura de São Paulo & 1 \\
\hline Scuola de psicoterapia ICLeS - Itália & 1 \\
\hline Sociedade Brasileira de Psicanálise de São Paulo & 1 \\
\hline Sociedade Brasileira de Psicologia Analítica & 1 \\
\hline Sociedade de Psicodrama de São Paulo & 1 \\
\hline Sociedade de Psicoterapias Analíticas Grupais do Estado de São Paulo & 6 \\
\hline Sociedade Internacional de Neuropsicanálise & 2 \\
\hline Sociedade Portuguesa de Grupoanálise & 2 \\
\hline Sociedade Psicanalítica de Porto Alegre & 2 \\
\hline Unisul & 1 \\
\hline Universidad de La Sabana & 1 \\
\hline Universidad Juárez del Estadi de Durango - México & 1 \\
\hline Universidad Santo Tomás - Bogotá & 2 \\
\hline Universidade Camilo Castelo Branco & 1 \\
\hline Universidade Católica de Brasília & 3 \\
\hline Universidade Católica de Goiás & 5 \\
\hline Universidade Católica de Pelotas - RS & 1 \\
\hline Universidade Católica de Pernambuco & 4 \\
\hline Universidade da Amazônia (UNAMA) & 1 \\
\hline Universidade de Brasília & 3 \\
\hline Universidade de Franca & 2 \\
\hline Universidade de Las Villas (Cuba) & 1 \\
\hline Universidade de São Paulo & 8 \\
\hline Universidade de São Paulo - Ribeirão Preto & 1 \\
\hline Universidade de Taubaté & 1 \\
\hline Universidade Estadual de Campinas & 5 \\
\hline Universidade Estadual de Londrina & 7 \\
\hline Universidade Estadual do Ceará & 2 \\
\hline Universidade Estadual do Mato Grosso do Sul & 2 \\
\hline Universidade Estadual do Rio de Janeiro & 1 \\
\hline Universidade Estadual Paulista - Assis & 3 \\
\hline
\end{tabular}


Quadro 1 - relação de instituições dos autores dos estudos (ordem alfabética de instituição; continuação).

\begin{tabular}{|c|c|}
\hline Instituições & $\begin{array}{c}\mathbf{N}^{0} \text { de } \\
\text { autores }\end{array}$ \\
\hline Universidade Estadual Paulista - Bauru & 1 \\
\hline Universidade Federal da Bahia & 1 \\
\hline Universidade Federal de Goiás & 1 \\
\hline Universidade Federal de Itajubá & 1 \\
\hline Universidade Federal de Minas Gerais & 1 \\
\hline Universidade Federal de Pernambuco & 1 \\
\hline Universidade Federal de Sergipe & 1 \\
\hline Universidade Federal de Uberlândia & 5 \\
\hline Universidade Federal do Ceará & 2 \\
\hline Universidade Federal do Pará & 1 \\
\hline Universidade Federal do Paraná & 1 \\
\hline Universidade Federal do Rio de Janeiro & 1 \\
\hline Universidade Federal do Rio Grande do Norte & 7 \\
\hline Universidade Federal do Rio Grande do Sul & 3 \\
\hline Universidade Federal do Vale do São Francisco & 1 \\
\hline Universidade Luterana do Brasil & 2 \\
\hline Universidade Luterana do Brasil - Gravataí & 2 \\
\hline Universidade Luterana do Brasil - Torres - RS & 1 \\
\hline Universidade Metodista & 2 \\
\hline Universidade Nova Lisboa & 1 \\
\hline Universidade Paris V - René Descartes & 2 \\
\hline Universidade Paulista & 1 \\
\hline Universidade Presbiteriana Mackenzie & 5 \\
\hline Universidade Sagrado Coração de Bauru & 1 \\
\hline Universidade Santo Amaro & 1 \\
\hline Universidade São Francisco & 2 \\
\hline Não Identificada & 7 \\
\hline
\end{tabular}

\section{Sexo dos Autores}

Outra questão analisada foi o sexo dos autores dos estudos. Ao todo, foram 205 autores levantados, dentre eles $65 \%$ foram do sexo feminino, $32 \%$ do masculino e houve ainda a categoria de autores cujo sexo não foi identificado (3\%), como é o caso de Samaniego, C. e Alkill Passalilcqua (SLAPAK; PASSALILCQUA; CERVONE, 2002), ambos autores estrangeiros.

Com esse resultado foi traçado o perfil de sexo dos autores das produções aqui analisadas, que se caracterizou como predominantemente feminino. Embora tenham sido observadas mudanças nos últimos anos no sentido de mais homens fazerem essa opção profissional, o fato é que, assim como ocorre com a Enfermagem e com o Serviço Social, a Psicologia ainda é praticada por maior número 
de mulheres na realidade brasileira. Esses dados corroboram essa característica cultural e também sinalizam uma outra característica da produção científica, de modo geral, nas últimas três décadas: as mulheres têm produzido mais, comparativamente a períodos históricos anteriores.

\section{Estratégias de AVALIAÇão PSICOLÓGICA}

Quanto às estratégias de avaliação psicológica utilizadas nos estudos, as entrevistas foram empregadas em nove trabalhos $(43,0 \%)$, o que mostrou um resultado não significante $[\chi 2(3, N=21)=6,98, p<0,05])$, seguidas das Escalas $(n=7$; $33,0 \%)$ e dos Testes Psicológicos $(\mathrm{n}=4 ; 19,0 \%)$, no total de 14 artigos que relatavam o uso de alguma estratégia. O uso do questionário também foi observado $(\mathrm{n}=1 ; 5,0 \%)$. Deste montante, em alguns foram usadas mais de uma estratégia, por exemplo, no estudo de Belle e Caminha (2005), três escalas compuseram o instrumental de pesquisa. Em outros casos, foram usados, num único estudo, testes e entrevistas (SILVA; ROSA; PAEGLE, 2004).

A título de ilustração, as estratégias padronizadas de avaliação psicológica utilizadas foram as seguintes: a) Testes: Teste da Figura Humana; Child Behavior Checklist (Ackenbach); Rorschach; e Teste de Relações Objetais de Philipson; b) Escalas: Escala Rutgers de Progresso em Psicoterapia; Escala Diagnóstica Adaptativa Operacionalizada (EDAO - R); Escala de Transtorno de Déficit de Atenção e Hiperatividade - para professores; Short-Form Alcohol Dependence Data; Escala de Avaliação do Craving; MTA (SNAP) - IV (sigla não especificada no artigo); e Protocolo de Transtorno de Déficit de Atenção e Hiperatividade - para pais.

Esses dados, juntamente com as outras freqüências observadas quanto ao uso das demais estratégias de avaliação psicológica utilizadas pelos autores dos trabalhos, sinalizam pequeno interesse pela pesquisa com amparo no uso dessas ferramentas metodológicas, informação que parece estar relacionada com o fato de que a maioria da produção científica, nesta amostra, trata-se de estudos teóricos.

Outra questão importante, sinalizada por esse achado, diz respeito à peculiaridade existente por parcela de psicoterapeutas, no sentido de entenderem que instrumentos padronizados e/ou de algum modo diretivos podem comprometer suas avaliações ocorridas no contexto de uma psicoterapia, em especial naquelas de orientação psicodinâmica. Nesse sentido, vale lembrar que esse enfoque teórico foi o mais frequente nos estudos que compuseram a amostra desta pesquisa.

\section{CONSIDERAÇÕeS FINAIS}

O número de produções científicas indexado na base eletrônica de dados PePsic teve elevado crescimento desde 1998, com concentração de maior número de artigos no ano de 2005 e uma queda na quantidade de publicações relativa aos anos de 2006 a 2007. Porém, esse fato ainda não pode ser considerado efetivo, já que têm sido constatados intervalos de até três anos entre a aceitação de um determinado artigo por um editor, até o momento de sua publicação. 
Os autores são em sua maioria do sexo feminino. Esse dado é importante diante do fato de que a Psicologia é uma profissão historicamente feminina na realidade espelhada por produções divulgadas por periódicos eminentemente brasileiros. Porém, outro dado é que o papel da mulher na produção do conhecimento científico tem crescido de modo significativo nas duas últimas décadas, mesmo em outras áreas, antes consideradas masculinas. Dos tipos de trabalhos considerados, houve maior número daqueles teóricos, seguidos por empíricos, mostrando um crescente interesse da comunidade científica por este último tipo de pesquisa.

Foi constatado que 5 dos periódicos divulgadores possuem $50,5 \%$ de toda a produção pesquisada, demonstrando uma maior concentração de produção científica sobre psicoterapias, dentre esses, um forte indício de serem os maiores divulgadores da área. O periódico do Conselho Federal de Psicologia (Psicologia: Ciência e Profissão) foi o que obteve maior número de publicações sobre psicoterapia. Esse dado aponta para uma característica conhecida e pesquisada pelo próprio Conselho, referente à área clínica como sendo aquela mais seguida entre profissionais no Brasil.

O enfoque teórico psicanalítico teve maior predominância nos estudos, seguido do cognitivo e comportamental. Esses são dois dos referenciais que mais influenciam estudos e pesquisas sobre psicoterapia, em diversas culturas, incluindo o Brasil e outros países na América Latina.

Houve predomínio significante de estudos que enfocaram a infância, e, em contrapartida, um menor número de produções científicas que se preocuparam com as outras etapas do desenvolvimento. Dentre essas, ressalta-se a parca divulgação sobre processos desenvolvidos com idosos, algo que tende a ser modificado em breve, dado o envelhecimento progressivo da população, bem como os ganhos recentes adquiridos por pessoas velhas em termos de longevidade.

Sobre as psicopatologias, não houve grande interesse por parte dos autores dos artigos em analisar o processo patológico, uma vez que a expressão dos dados corresponde a um número pequeno em relação ao montante de trabalhos analisados, indicando uma tendência dos pesquisadores a não enfocar a patologia como área de estudo. Entretanto, são necessários avanços nesta área, uma vez que as doenças mentais são muitas vezes questões de Saúde Pública e seus índices são também alarmantes na população brasileira e mundial. Outro ponto que pode ser destacado a esse respeito é aquele que indica ser a "psicopatologia" uma terminologia oriunda do paradigma médico, o que nem sempre encontra ressonância entre psicólogos e psicoterapeutas.

Vale ressaltar que estudos relativos a psicopatologias específicas continuam a despontar como tendências e perspectivas em publicações na área da psicoterapia, já que disponibilizam descrições sobre as principais teorias, suas bases de evidências, "[...] princípios práticos fundamentais e diretrizes para lidar com desafios difíceis", tal como observam Gabbard, Beck e Holmes (2007, p. 11). É sabido que ainda são marcantes as diferenças entre as produções estrangeiras e as nacionais nesse sentido, muitas delas facilmente visualizadas na 
produção ora considerada, e outras disponíveis em outros suportes de comunicação acadêmica, produzidos na realidade brasileira ou não (FRIEDMAN, 2009; SANTEIRO, 2005; TOLMAN, 2009).

A maior parte dos autores está afiliada a instituições brasileiras, as quais estão concentradas na região sudeste do país. E as estratégias de avaliação investigadas mostraram interesse esparso e não significante, pela Entrevista e Escala, ainda em número inexpressivo diante da quantidade de trabalhos analisados. Pode-se pensar que para um avanço na área das pesquisas em psicoterapia muito tenha que ser feito quanto aos instrumentos de medidas psicológicas, para que as informações e dados estudados tenham maior consistência e não se baseiem apenas em intuições (ROBINS; GOSLING; CRAIK, 1999).

Sem dúvida, pesquisar é necessário. É uma das formas possíveis de difundir o conhecimento, quando o produto deste processo vem a público. Analisar a produção científica "traz em si a dinâmica da descoberta" (PACHECO, 2005, p. 20) com a possibilidade de uma abertura para visualizar o panorama geral da área pesquisada, aqui delineada pela psicoterapia.

Este estudo pôde verificar um crescente aumento no interesse por pesquisar a psicoterapia, na base de dados PePsic, o que pode, ou não, ser confirmado nos próximos anos, diante de suas inúmeras formas. Porém, ainda é necessário fazer algo por uma pesquisa mais sistematizada, principalmente no âmbito nacional, onde ainda é necessário um crescimento dos estudos empíricos e validações de instrumentos pertinentes a esta prática, que aqui foi tido como incipiente.

Num momento em que a psicoterapia é tomada como objeto de atenção especial pelo Sistema Conselhos e no qual novas formas de atenção psicoterapêutica, geradas sob a luz de novos paradigmas, são irrevogáveis, discutir o que se produz sobre psicoterapias e por psicoterapeutas é movimento essencial ao ganho de robustez do próprio fazer psicoterapêutico. É possível considerar, também, que no momento em que as interfaces entre Psicologia e Informática podem ser progressivamente úteis ao psicoterapeuta, os antigos casos clínicos podem dividir espaço com os "casos das bases de dados".

Tão relevante quanto entender as expressões humanas em settings psicoterapêuticos, seria entender as expressões dos psicoterapeutas direcionadas ao seu público leitor: estudantes em formação, pesquisadores e colegas de trabalho. Nesse sentido, esse relato de pesquisa introduz questões ao leitor e convida a que psicoterapeutas estejam atentos ao que se diz sobre psicoterapia, para além das variáveis aqui atendidas e para além da PePsic. 


\section{REFERÊNCIAS}

ALMEIDA, C. C.; COSTA, G. O.; GOMES, K. V. Segredos e conflitos familiares: um estudo de caso. Vínculo, v. 6, n. 1, p. 88-99, jun. 2009.

BELLE, A. H.; CAMINHA, R. M. Grupoterapia cognitivo-comportamental em crianças com TDAH: estudando um modelo clínico. Revista Brasileira de Terapia Cognitiva, v. 1, n. 2, p. 103-114, dez. 2005.

BRUM, E. H. M. A depressão materna e suas vicissitudes. Psyche. São Paulo, v. 10, n. 19, p. 95-108, dez. 2006.

CAMPOS, A. P. S.; CURY, V. E. Atenção psicológica clínica: encontros terapêuticos com crianças em uma creche. Paidéia. Ribeirão Preto, v. 19, n. 42, p. 115-121, abr. 2009.

CAÑETE, M. C. V.; VITALLE, M. S. S.; SILVA, F. C. Anorexia nervosa: estudo de caso com uma abordagem de sucesso. Fractal, Rev. Psicol. [online], v. 20, n. 2, p. 377-386, jul./dez. 2008.

COELHO, L. S. G. Aprendizagem vicária de treino de toalete através de filme de animação: estudo de caso em ludoterapia comportamental. Psicologia: Ciência e Profissão, v. 28, n. 4, p. 846-861, dez. 2008.

COSTA, M. I. M.; DIAS, C. M. S. B. A prática da psicoterapia infantil na visão de terapeutas nas seguintes abordagens: psicodrama, Gestalt terapia e centrada na pessoa. Estud. psicol. Campinas, v. 22, n. 1, p. 43-51, mar. 2005.

D’EL REY, G. J. F.; PACINI, C. A. Terapia cognitivo-comportamental da fobia social: modelos e técnicas. Psicologia em Estudo, v. 11, n. 2, p. 269-275, ago. 2006.

FINKEL, L. A. O lugar da mãe na psicoterapia da criança: uma experiência de atendimento psicológico na saúde pública. Psicologia: Ciência e Profissão, v. 29, n. 1, p. 190-203, mar. 2009.

FIORINI H. J. Teoria e Técnica de Psicoterapias. Trad. de Carlos Sussekind. 9. ed. Rio de Janeiro: Francisco Alves. Título do original: Teoría y técnica de psicoterapias, 1991.

FRIEDMAN, M. Transtorno de estresse agudo e pós-traumático. 4. ed. Trad. de Marina Fodra. Porto Alegre: Artmed, 2009. 
GABBARD, G. O.; BECK, J. S.; HOLMES, J. Compêndio de psicoterapia de Oxford. Trad. de Magda F. Lopes e Ronaldo C. Costa. Porto Alegre: Artmed, 2007.

GRETHER, I. G.; HASSENE, D. R. Psicoterapia psicanalítica breve. Revista de Psiquiatria do Rio Grande do Sul, v. 29, n. 2, p. 241-242, ago. 2007.

GULASSA, D. Vínculo e confiança em atendimento psicoterapêutico psicodramático grupal com presidiários. Psicologia: Ciência e Profissão, v. 27, n. 2, p. 332-341, jun. 2007.

MESSER, S.B.; WATCHEL, P.L. The contemporary psychotherapeutic landscape: Issues and prospects. In:

.Theories of psychotherapy: evolution and current status, Washington, DC: APA Books, 1997. p. 1-38.

MOTTA, L. F.; SANTOS, M. A. Grupoterapia breve de múltiplos casais em hospital-dia. Paidéia. Ribeirão Preto, v. 12, n. 22, p. 69-81, 2002.

NEUBERN, M. Histórias que (não) curam: sobre narrativas em hipnose clínica. Psicologia: Ciência e Profissão, v. 24, n. 3, p. 58-65, set. 2004.

NEUBERN, M. Hipnose e psicologia clínica: retomando a história não contada. Psicologia: Reflexão e Crítica, v. 19, n. 3, p. 346-354, 2006.

ODDONE, H. R. B. Suicídio e psicoterapia: uma visão gestáltica. Estudos e Pesquisas em Psicologia, v. 5, n. 1, p. 165-167, jun. 2005.

OLIVEIRA FILHO, R. S.; HOCHMAN, B.; NAHAS, F. X.. Fomento à publicação científica e proteção do conhecimento científico. Acta Cirurgica Brasileira, v. 20, suppl. 2, p. 35-39, 2005.

PACHECO, E. M. C. Produção Cientifica e Avaliação Psicológica. In: Metaciência e Psicologia. Campinas: Alínea, 2005, p. 7-33.

PAPALIA, D. E.; OLDS, S. W.; FELDMAN, R. D. Desenvolvimento Humano. Trad. de Daniel Bueno. 8. ed. Porto Alegre: Artmed, 2006.

ROBINS, R. W.; GOSLING, S. D.; CRAIK, K. H. An empirical analysis of trends in psychology. American Psychologist, v. 54, n. 2, p. 117-128, 1999.

RODRIGUES, H. J. L. F.; BRITO, A. L. Ano da psicoterapia: textos geradores. Brasília: Conselho Federal de Psicologia, 2009. 
SADOCK. B. J.; SADOCK, V. A. Compêndio de Psiquiatria: ciência do comportamento e psiquiatria clínica. Trad. de Claudia Dornelles et al. 9. ed. Porto Alegre: Artmed, 2007.

SAKAMOTO, C. K. Foco e estratégia da supervisão clínica em psicoterapia breve. Cadernos de Psicopedagogia, v. 6, n. 10, 2006. Disponível em: <http://pepsic. bvs-psi.org.br/scielo.php?script=sci_arttext\&pid=S1676-10492006000100003$\& \operatorname{lng}=$ pt\&nrm=>. Acesso em: 10 ago. 2009.

SANTEIRO, T. V. Psicoterapias breves psicodinâmicas: produção científica em periódicos nacionais e estrangeiros (1980/2002). 2005. Tese (Doutorado)-Pontifícia Universidade Católica de Campinas, Campinas, 2005.

SANTEIRO, T. V. Psicoterapia breve psicodinâmica preventiva: pesquisa exploratória de resultados e acompanhamento. Psicologia em Estudo, v.13, n.4, p.761770, dez. 2008.

SANTOS, M.; ZASLAVSKY, J. Pesquisando conceitos e tendências em psicoterapia e psicanálise. Revista Brasileira de Psicanálise, v.41, n.2, p.115-124, jun. 2007.

SEI, M. B. Abrindo espaço para o ser: Winnicott e a ludoterapia no contexto da violência familiar. Psyche. São Paulo, v.12, n.22, p.199-214, jun. 2008.

SILVA, J. V. V.; ROSA, J. T.; PAEGLE, I. C. Contribuições do Teste de Relações Objetais de Phillipson para o diagnóstico do funcionamento mental de pacientes com transtorno de pânico. Psic, v.5, n.1, p.48-65, jun. 2004.

SLAPAK, S.; PASSALILCQUA, A.; CERVONE, N. Cambio psiquico: técnicas e instrumentos de evaluación aplicados a niños que realizan psicoterapia psicoanalitica grupal. Psic, v.3, n.1, p.86-97, jun. 2002.

SOUZA, M. M.; TEIXEIRA, R. P. O que é ser um "bom” psicoterapeuta? Aletheia, n.20, p.45-54, dez. 2004.

STONE. M. H. História da Psicoterapia. In: - Psicoterapia de orientação analítica: fundamentos teóricos e clínicos. 2. ed. Porto Alegre: Artmed, 2005. p. 23-42.

TEIXEIRA, M. C. T. V.; GONCALVES, F. L. Relato sobre o XIV Encontro Brasileiro de Psicoterapia e Medicina Comportamental. Psicologia: Teoria e Prática, v. 7, n. 2, p. 243-246, dez. 2005.

TOLMAN, A. Depressão em adultos. 3. ed. Trad. Sandra Mallmann. Porto Alegre: Artmed, 2009. 
Fabiana Pessini Pinto; Tales Vilela Santeiro; Fabiola Ribeiro de Moraes Santeiro

WEISSMAN, M. M.; MARKOWITZ, J. C.; KLERMAN, G. L. Psicoterapia interpessoal: guia prático do terapeuta. Trad. de Sandra M. Mallmann da Rosa. Porto Alegre: Artmed, 2009.

WITTER, C.; BURITI M. A.; WITTER, G. P. (Orgs.). Problemas psicossociais: análise de produção. Guararema, SP: Anadarco, 2007.

Recebido em: setembro de 2009

Aceito em: maio de 2010 